\title{
Fluid transport in open-cell polymeric foams: effect of morphology and surface wettability
}

\author{
Boran Zhao ${ }^{1} \cdot$ Gabriel Gedler $^{1} \cdot$ Ica Manas-Zloczower $^{2} \cdot$ Stuart J. Rowan $^{2,3} \cdot$ Donald L. Feke $^{1}$ (D)
}

Received: 18 October 2019 / Accepted: 6 January 2020 / Published online: 10 January 2020

(c) Springer Nature Switzerland AG 2020

\begin{abstract}
Fluid transport behavior inside polymer foams (polyHIPEs) templated from high-internal-phase emulsions (HIPEs) has been studied. Morphological properties of the foams were controlled through shearing their precursor emulsions for varying durations. Two types of flow, Darcy flow through the filled foam, and capillary imbibition into unfilled foams, have been studied. From permeability measurement, the size of the interconnections (windows) between the voids of the polyHIPE was found to provide the appropriate characteristic length to describe Darcy flow. For capillary imbibition, liquid/foam interaction effects were studied under two wetting conditions: silicone oil in which the oil readily spreads (at a vanishing contact angle) on the struts within the foam; and, using water and foams coated with various amount of surfactant where the liquid only partially wets the surface. For capillary imbibition, the dominant morphological feature was also the window size when the internal surfaces of the foam have a macroscopically uniform wettability for the liquid. The Lucas-Washburn analysis was found to well describe imbibition rates in this case. However, when the internal surfaces of the foam have a non-uniform wettability (due to a non-uniform deposition of surfactants), the concentration and distribution of surfactants plays a large role in determining imbibition kinetics.
\end{abstract}

Keywords High-internal phase emulsions (HIPEs) · Polymer foams · Morphology · Spontaneous imbibition · Wettability

\section{Introduction}

PolyHIPEs are open-cell polymeric foams fabricated by polymerizing the continuous phase of high internal phase emulsions (HIPEs). Because the dispersed phase within HIPEs exceeds $74 \%$ by volume, polyHIPE foams not only have high porosity but also have good interconnectivity (via windows) between neighboring voids. Recent studies have demonstrated the potential of polyHIPEs as scaffolds in tissue engineering $[18,19,23]$, for oil-spill recovery [31], and as supports or membranes in separations and absorption [21]. Typical applications of polyHIPEs involve the transport of fluid within the porous structure, which depends on two families of parameters. One relates to the morphological properties of polyHIPEs (i.e., porosity, void size and interconnect/window size and size distribution) while the other relates to the interaction of polyHIPE material with the fluid (the wetting of the material by the fluid) $[13,20,25]$. For separations-related applications, morphological properties are usually more important than interfacial interactions and wettability because the polyHIPE is typically saturated with fluid, and the flow will be one phase. However, for applications that involve two-phase flow such as in oil recovery and absorbing where there may be multiple fluid phases, both the polyHIPE morphology and polymer-fluid interactions matter.

It has been shown that the morphological properties of the polyHIPEs can be controlled by the HIPE emulsification

Donald L. Feke, donald.feke@case.edu | 'Department of Chemical and Biomolecular Engineering, Case Western Reserve University, Cleveland, OH 44106, USA. ${ }^{2}$ Department of Macromolecular Science and Engineering, Case Western Reserve University, Cleveland, $\mathrm{OH} 44106$, USA. ${ }^{3}$ Institute for Molecular Engineering, University of Chicago, Chicago, IL 60637, USA. 
process [5]. For example, in a water-in-oil emulsion, the effect of water-to-oil phase ratio [16], emulsifier content [28] and shearing conditions on the final polyHIPE foam have been investigated in various studies. The polyHIPE structure can be further tuned after polymerization by methods like hyper-crosslinking [24]. Although polyHIPEs with various structures have been fabricated, only a few studies [30] investigated fluid transport in the Darcy flow regime and therefore understanding of the influence of polyHIPE morphological properties on fluid transport behavior in other than Darcy flow (e.g., in capillary imbibition) is incomplete. Furthermore, to our best knowledge the effect of polyHIPE surface wettability on fluid transport behavior has not been reported. Elucidating these fundamental aspects of the transport behavior within polyHIPEs forms the objectives of this study.

Through controlling morphological parameters of polyHIPEs, this paper provides fundamental understanding of the fluid flow behavior in polyHIPEs in Darcy flow and capillary imbibition in which the Washburn-Lucas analysis is typically applied to investigate the fine structure of porous medium [14]. In addition, the interaction of the polyHIPE with infiltrating liquids was controlled through the use of emulsifiers and thus capillary imbibition under wetting or partial wetting conditions were investigated.

\section{Experimental}

\subsection{Materials}

The oil phase for the emulsions was prepared from a mixture of 2-ethylhexyl acrylate (2-EHA), 2-ethylhexyl methacrylate (2-EHMA) and ethylene glycol dimethacrylate (EGDMA) (which acts as the crosslinker) in a ratio of 2:2:1. The monomers were purchased from Sigma-Aldrich and used as received. A 7:1 mixture of nonionic (polyglycerol succinate, PGS) and ionic (ditallow dimethyl ammonium sulfate, DTDMAMS) surfactants was used at a total concentration of $7 \mathrm{wt} \%$. Deionized water, purified to a conductivity below $15 \mu \mathrm{S} / \mathrm{m}$ was used for the aqueous phase. Sodium chloride $(\mathrm{NaCl}, 2 \mathrm{wt} \%)$ was added to the aqueous phase, as was the initiator sodium persulfate (NaPS, $0.33 \mathrm{wt} \%)$. The initiator was purchased from Sigma-Aldrich and used as received. Silicone oils for the imbibition tests were kindly supplied by Dow-Corning and used as received.

\subsection{Methods}

\subsubsection{Emulsion preparation}

The emulsification process was described in detail in a previous work [10]. In brief, emulsions were prepared using a bench-top batch process in which the temperature of the system was maintained at $50^{\circ} \mathrm{C}$ using a heating jacket. The aqueous phase, preheated to $50^{\circ} \mathrm{C}$, was added into the oil phase dropwise by hand and mixed utilizing an overhead mixer. Emulsion samples were collected into centrifuge tubes and polymerized at $85^{\circ} \mathrm{C}$ for $4 \mathrm{~h}$. With the aim of varying the emulsion droplet size, the shearing time was varied from 5 to $30 \mathrm{~min}$. Since both the shearing time and aqueous-to-oil-phase ratio were varied, the samples were coded in the format RxxMxx where $\mathrm{R}$ indicates the aqueous-to-oil-phase ratio and $M$ gives the mixing time in minutes. For example, R19M10 represents the emulsion/ foam having an aqueous-to-oil-phase ratio of 19 prepared by mixing for $10 \mathrm{~min}$.

\subsubsection{Foam morphology}

Foam morphologies were observed using a JEOL JSM$6510 \mathrm{LV}$ scanning electron microscope (SEM). The voltage was adjusted between 15 and $30 \mathrm{kV}$ to get proper images for the window sizes and the void sizes separately. The samples were previously washed with deionized waterisopropanol-deionized water by Soxhlet extraction for $24 \mathrm{~h}$ each, and subsequently freeze-dried in order to avoid any deformation of the foam morphology. The samples were cut with a razor blade and were subsequently coated by depositing a thin layer of gold using a Hummer 6.2 Anatech Ltd. sputter system in a nitrogen atmosphere. To obtain information on the void and interconnecting window diameters, three SEM micrographs with different magnifications were used for each foam sample and the image processed using ImageJ software. At least 300 voids/windows were sampled for each micrograph. The void diameter was characterized in terms of both the number-average diameter $\left(D_{10}\right)$ and Sauter-mean diameter $\left(D_{32}\right)$ according to:

$D_{10}=\frac{\sum n_{i} d_{i}}{\sum n_{i}}$

$D_{32}=\frac{\sum n_{i} d_{i}^{3}}{\sum n_{i} d_{i}^{2}}$

where $d_{i}$ are the individual void/window diameters interpreted from the SEM micrographs and $n_{i}$ is the number of that specific void or window size in the micrograph. The Sauter-mean diameter gives more weight to the larger voids within the polyHIPE foams, and this measure is commonly used to characterize emulsions. The breadth of the distribution of void or window sizes in the polyHIPE is characterized by the polydispersity index $(P D I)$ defined by: 
$P D I=D_{32} / D_{10}$

In order to interpret SEM micrographs properly, it is necessary to employ a statistical correction because the twodimensional images of the voids being analyzed may show a cross-section at a random position relative to the center of the void. As shown in Fig. 1a, assuming a random cut with a distance $r_{i}$ from the center of the "sperical" void, the corresponding radius seen in the image is $r_{s}$. The assumption of a random cut through the radius promises the equal probability of $r_{i}$ between 0 and $R_{0}$, thus the expectation of the average radius from a random cut is given by:

$\mathrm{E}\left(r_{s}\right)=\frac{\int_{0}^{R_{0}} \sqrt{R_{0}^{2}-r_{i}^{2}} d r_{i}}{\int_{0}^{R_{0}} d r_{i}}=\frac{\pi}{4} R_{0}$

Thus, the most likely diameter of a void that appears in the SEM micrograph to have diameter $d_{S E M}$ is $d_{i}=4 d_{S E M} / \pi$.

The correction factor for the the window size is different from that for the void size. As shown in Fig. 1b asuming that the distribution of windows is isotropic around the void surface, the projection of a circular window onto the 2-D SEM image will be a ellipse and the projected area is given by:

$A_{\text {projection }}=\cos \theta A_{\text {window }}$

where $\cos \theta$ is defined as the ratio of minor and major axis of the ellipse and $\theta \in\left[0, \frac{\pi}{2}\right]$. The expectation of the window area is thus given by:
$\mathrm{E}\left(A_{\text {projection }}\right)=\mathrm{E}(\cos \theta) A_{\text {window }}$

$\mathrm{E}(\cos \theta)=\frac{\int_{0}^{\frac{\pi}{2}} \cos \theta d \theta}{\int_{0}^{\frac{\pi}{2}} d \theta}=\frac{2}{\pi}$

The window size is computed as the radius of the circle that has equivalent area to the window seen in the image. Thus, the most likely size of the window $\left(D_{w}\right)$ is related to the apparent window size $\left(D_{a p p, w}\right)$ from the SEM micrograph, by $D_{w}=D_{a p p, w} \sqrt{\pi / 2}$.

\subsubsection{Foam density}

The density of the various polyHIPE foams was measured according to a standard procedure (i.e. ISO 845) involving direct measurement of the mass and volume of the samples.

\subsubsection{Foam mechanical properties}

In order to determine the degree to which the polyHIPE deformed or compressed in the fluid flow experiments, the Young's modulus of the foams was measured from a stress-strain curve in uniaxial compression test using an Instron 1101 platform operated at $1 \mathrm{~mm} / \mathrm{min}$. Foam samples were cut into $25-\mathrm{mm}$ diameter, $10-\mathrm{mm}$ thick discs for mechanical testing. The modulus was extracted from the linear region of the stress-strain curve.
Fig. 1 Definition of geometric variable for computing the relationship between: $\mathbf{a}$ the radius of a void $\left(R_{0}\right)$ and the apparent radius $\left(r_{s}\right)$ seen in a SEM image and $\mathbf{b}$ a circular window on a void and its projection based on viewing angle

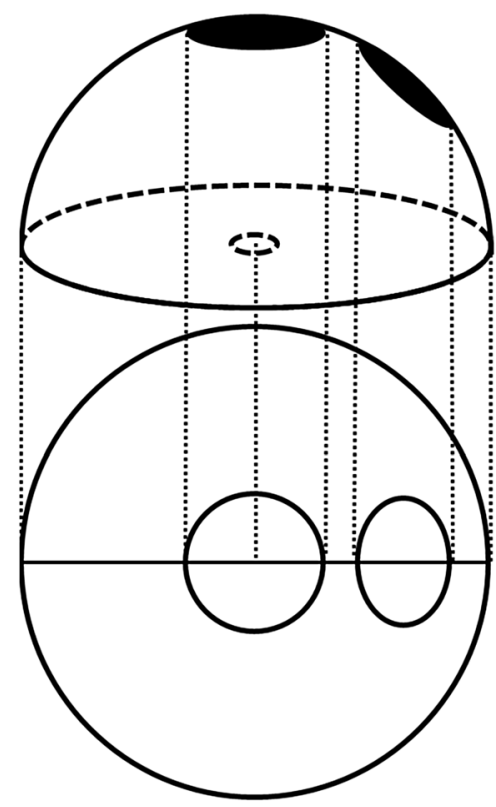

(b) 


\subsection{Permeability measurement}

The water flux passing through thin polyHIPE foams was measured under various applied pressure drops. For this purpose, polyHIPE foams were cut into thin (1-3 mm thick) discs. Samples to be tested were then clamped between a glass filtration set, as is depicted schematically in Fig. 2 . The bottom piece of the filtration set is a fritted-glass base (4.8 $\mathrm{mm}$ in thickness) and the top piece is a glass tube filled with water. The pressure drop across the foam was achieved by using a range of heights $(0.1-1.5 \mathrm{~m})$ of the water column in the tube and a funnel with a large cross-sectional area to maintain a constant water- column height. Water was replenished into the funnel at a certain time interval to maintain a uniform driving pressure. The effective cross-sectional area subjected to flow was equal to the fritted-glass base area, which was $2.2 \mathrm{~cm}^{2}$. At least three different pressure gradients were selected for each foam sample and data from three foams were averaged for each flow condition. The pressure applied to the foams was selected according to the foam compression stress-strain curve and under all the pressures the foam was compressed to no more than $5 \%$ strain. The foams were saturated with water throughout the process. Water fluxes were plotted against pressure drops across the foam

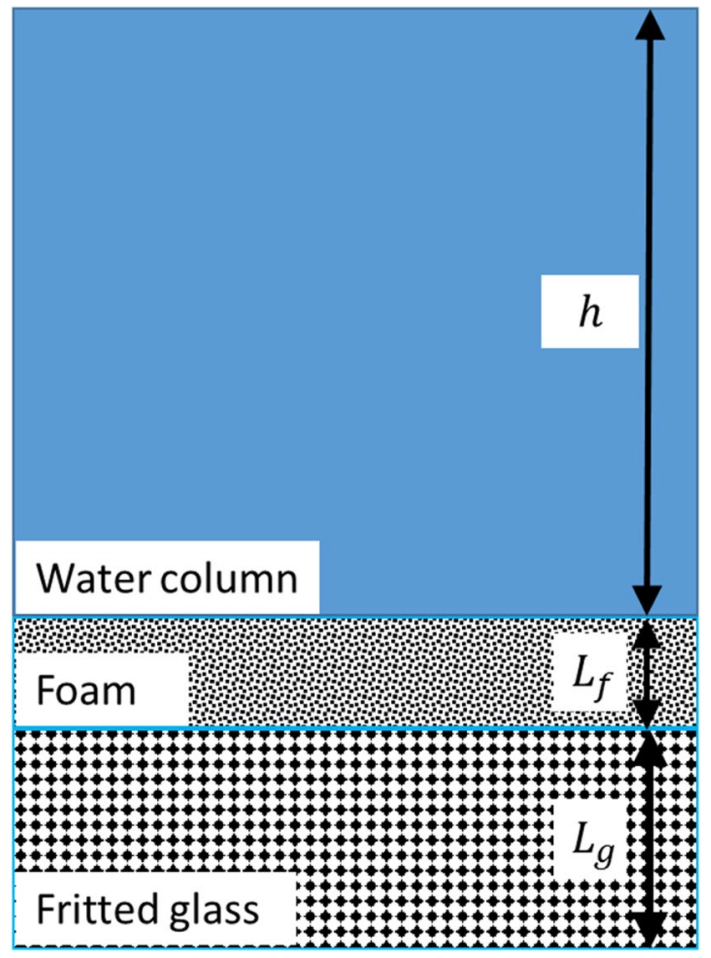

Fig. 2 Schematic illustration of the experiment to measure permeability and the slope of the curve was extracted for the permeability calculation.

The overall permeability for the complete experimental assembly is given by Eq. 8 [6] where $\dot{V}$ is the observed volumetric flow rate, $A$ is the flow cross section area, $K_{t}$ is the overall permeability, $\Delta P$ is the pressure drop across the foam, $\mu$ is the liquid viscosity and $L_{t}$ is the overall length of the flow path through the foam and fritted-glass support.

$\dot{V}=\frac{A K_{t} \Delta P}{\mu L_{t}}$

The permeability for the fritted-glass member $\left(K_{g}\right)$ was measured in a separate experiment without foam present, which resulted in $K_{g}=132.0 \times 10^{-13} \mathrm{~m}^{2}$. This value is at least one order-of-magnitude larger than the foam permeability $\left(K_{f}\right)$. In an experiment involving foam, since the flow passes through the foam and fritted-glass support in series, the various permeabilities are related to the known values of foam thickness $\left(L_{f}\right)$ and glass thickness $\left(L_{g}\right)$ through:

$\frac{L_{t}}{K_{t}}=\frac{L_{f}}{K_{f}}+\frac{L_{g}}{K_{g}}$

Once $K_{f}$ is determined, the flow channels within the polyHIPE can be associated with a characteristic hydrodynamic radius $(r)$ calculated from [12]

$K_{f}=\frac{\emptyset r^{2}}{8 \tau}, \tau=\frac{\emptyset}{1-(1-\emptyset)^{1 / 3}}$

where $\emptyset$ is the porosity and $\tau$ is the tortuosity factor calculated from the porosity [1].

Since the foams were fully saturated with the DI water before and during the test, the results of the permeability experiments provides information on only the foam morphological properties.

\subsection{Spontaneous imbibition measurement}

The uptake of liquid into polyHIPE foams by capillary action was monitored using a home-built setup illustrated schematically in Fig. 3. PolyHIPE foams were cut into 25-mm diameter and 10-mm (or 5-mm for some samples) height discs. These were placed into open-ended plastic (machined from 50-ml centrifuge tubes) sleeves and then held facing downward vertically to a stand resting on a balance (Mettler Toledo). Subsequently, the bottom of the foam sample was brought into contact with the pool of test liquid. The accumulated weight of the penetrating liquid was monitored by the balance and recorded by computer. The raw liquid-uptake data was corrected for buoyancy effect from the reservoir of test liquid. The 


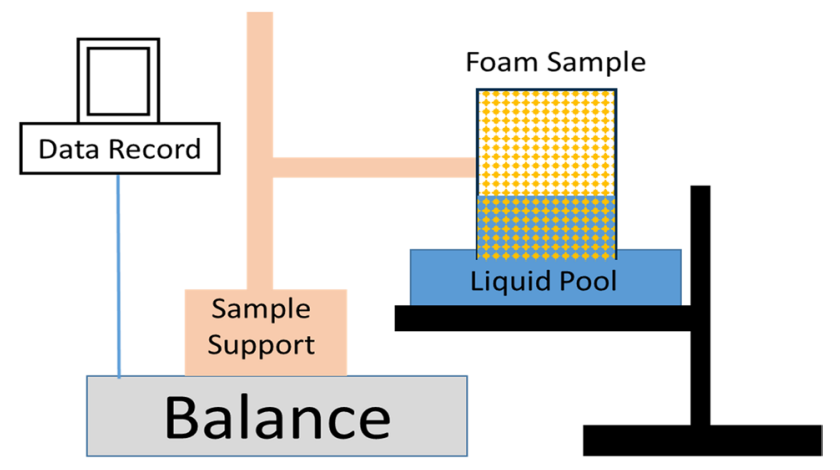

Fig. 3 Schematic illustration of the experiment to measure spontaneous imbibition

imbibition weight was then plotted against the square root of time and the slope of the linear region from the curve was extracted to calculate the characteristic capillary diameter. For the silicone oil imbibition test, the foams were washed separately with DI water and isopropyl alcohol (IPA) for $24 \mathrm{~h}$ by Soxhlation to strip off any residual electrolyte and surfactants and then dried in convection oven at $65^{\circ} \mathrm{C}$ for $24 \mathrm{~h}$. For water imbibition tests, additional surfactant was added to the polyHIPE foams by soaking them with various concentration of PGS (in IPA) solutions followed by squeezing. This process was repeated three times and subsequently the squeezed foams were dried under vacuum at room temperature. The physical properties of the silicone oils used in this study were measured and compared with the manufacturer-supplied data.

\section{Results}

\subsection{Variation of foam morphology}

The morphology of the foams results from the competition between the mechanisms of droplet breakup and coalescence within the parent HIPE, both of which are affected by the balance between the shearing forces applied to the suspension and interfacial effects which dictate the stability of the suspension $[11,26]$. In practice the emulsion morphology could be tuned by varying the strength of shear applied. However, in this study, droplet size was controlled by varying the mixing time during emulsification. Also, it was found that the polydispersity in droplet size was reduced by increased shearing time.

Figure 4 shows typical morphology of polyHIPE foams templated from the HIPEs. As shown in Table 1, polyHIPE foams with average void sizes from 36.2 to $13.9 \mu \mathrm{m}$ were achieved. The average window size was also found to be reduced from 9.4 to $4.2 \mu \mathrm{m}$ as the shearing time for the emulsion was increased. The ratio of average void to window diameter remained very similar for all the foams at around 3.3.

\subsection{Darcy flow through polyHIPE foams}

A typical set of results for the water flux as a function of pressure drop across the foam is shown in Fig. 5. A similar linearity was found for all foams tested in this work, and thus Darcy's law applies to the polyHIPE foams.

Table 2 summarizes the measured foam permeability and the hydrodynamic diameter $\left(D_{K}\right)$ calculated from the permeability through Eq. 10. Also included in Table 2 is information on the window size within the foam as determined from the SEM micrograph analysis $\left(D_{32, w}\right)$. Note the excellent agreement between $D_{K}$ and $D_{32, w}$. This consistency confirms the validity of SEM image analysis method used in this work and demonstrates that the critical morphological parameter controlling Darcy flow in polyHIPEs is the window size.

\subsection{Spontaneous imbibition in polyHIPEs}

The imbibition of liquid into a porous medium is characterized by a penetration distance $h$ that scales with the square root of time $\langle h\rangle \sim t^{1 / 2}$ according to the wellknown Lucas-Washburn (L-W) law [17, 27] (although Bell and Cameron [2] studied this phenomenon earlier). In the
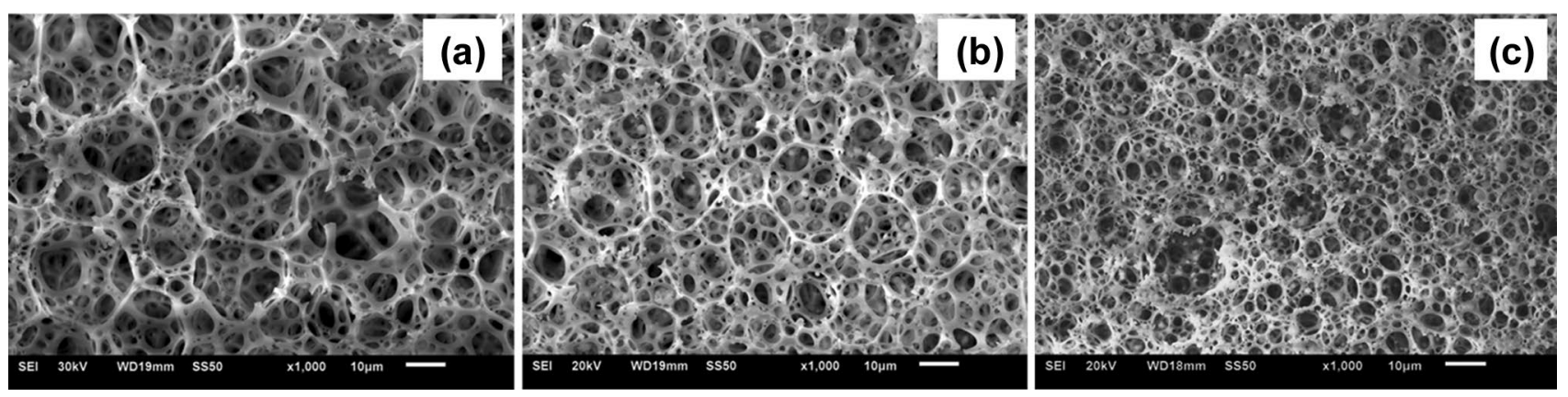

Fig. 4 Typical polyHIPE morphologies for R19 foams: a $M=1 \min , \mathbf{b} M=5 \min , \mathbf{c} M=30 \min$ 
Table 1 Processing parameters, morphological properties and mechanical properties for polyyHIPE foams

\begin{tabular}{lccccccc}
\hline Sample & $t(\mathrm{~min})$ & $D_{32, v}(\mu \mathrm{m})$ & PDI & $D_{32, w}(\mu \mathrm{m})$ & PDI & $E(\mathrm{kPa})$ & $S(\mathrm{kPa})$ \\
\hline R19M05 & 5 & $36.2 \pm 2.3$ & 1.26 & $9.4 \pm 0.7$ & 1.22 & $277 \pm 11$ & $29.4 \pm 1.0$ \\
R19M10 & 10 & $22.4 \pm 3.4$ & 1.20 & $6.9 \pm 0.5$ & 1.21 & $256 \pm 35$ & $32.7 \pm 0.4$ \\
R19M15 & 15 & $16.5 \pm 0.9$ & 1.12 & $6.4 \pm 0.6$ & 1.07 & $293 \pm 7$ & $34.2 \pm 0.3$ \\
R19M30 & 30 & $13.9 \pm 0.9$ & 1.18 & $4.2 \pm 0.4$ & 1.16 & $291 \pm 15$ & $37.3 \pm 0.2$ \\
\hline
\end{tabular}

$t$ emulsion mixing time, $D_{32, v}$ Sauter-mean diameter of voids, $D_{32, w}$ Suter-mean diameter of windows, $P D /$ polydispersity index, $E$ Young's modulus of the dry foam, $S$ yield strength of the dry foam

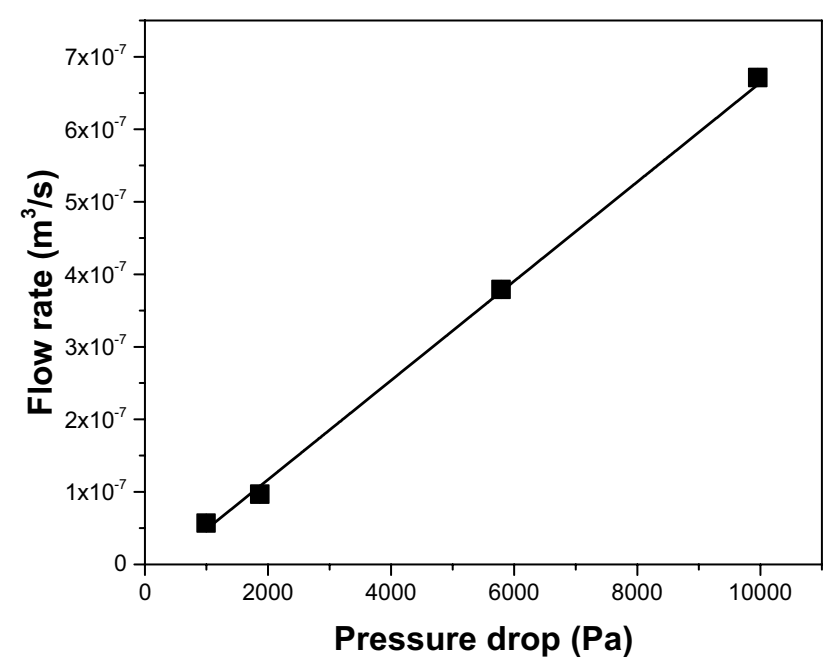

Fig. 5 Typical Darcy flow results. The foam sample was R10M10

Table 2 Characteristic hydrodynamic diameters from the permeability tests $\left(D_{K}\right)$ and window size by SEM analysis $\left(D_{32, w}\right)$

\begin{tabular}{lccc}
\hline Foam & $\begin{array}{l}\text { Permeability } \\
\left(\times 10^{-13} \mathrm{~m}^{2}\right)\end{array}$ & $D_{K}(\mu \mathrm{m})$ & $D_{32, w}(\mu \mathrm{m})$ \\
\hline R10M05 & $16.9 \pm 0.5$ & $9.2 \pm 0.4$ & $9.4 \pm 0.7$ \\
R19M10 & $7.5 \pm 0.4$ & $6.1 \pm 0.2$ & $6.9 \pm 0.5$ \\
R19M15 & $4.8 \pm 0.2$ & $5.2 \pm 0.3$ & $6.4 \pm 0.6$ \\
R19M30 & $3.0 \pm 0.3$ & $3.8 \pm 0.2$ & $4.2 \pm 0.4$ \\
\hline
\end{tabular}

present work, two scenarios were studied. One is the case of perfect wetting in which the liquid (silicone oil) interacts well with the struts within the polyHIPE, while the other is the partial-wetting case in which the wetting of the liquid (water) on the struts of the polyHIPE is controlled by coating with a surfactant.

A scaling analysis demonstrates the expected applicability of the Lucas-Washburn ( $L-W$ ) analysis to the imbibition process. The $L-W$ analysis has been reported to be inaccurate when either gravity [15] or fluid inertia plays an important role [7] in the imbibition process. The dimensionless groups relevant to capillary imbibition are defined in Eqs. 11-14. Table 3 shows definitions
Table 3 Parameters used in the scaling analysis

\begin{tabular}{ll}
\hline Physical properties & Characteristic value \\
\hline Capillary diameter $(D)$ & $10 \mu \mathrm{m}$ \\
Capillary length $(L)$ & $1 \mathrm{~cm}$ \\
Liquid density $(\rho)$ & $961.2 \mathrm{~kg} / \mathrm{m}^{3}$ \\
Liquid viscosity $(\mu)$ & $0.106 \mathrm{~Pa} \mathrm{~s}$ \\
Liquid surface tension $(\gamma)$ & $21 \mathrm{mN} / \mathrm{m}$ \\
Infiltration velocity $(v)$ & $1 \mathrm{~mm} / \mathrm{s}$ \\
Acceleration of gravity $(g)$ & $9.8 \mathrm{~m} / \mathrm{s}^{2}$ \\
\hline
\end{tabular}

and estimates of the relevant physical parameters. The Bond number $\left(B_{o}\right)[8]$ represents the relative importance of gravity to surface tension and was calculated to be on the order of $10^{-5}$ in the current experiment setup. The Weber number $\left(W_{e}\right)$ represents the relative importance of inertia to surface tension and is calculated to be on the order of $10^{-7}$. The critical radius for inertia to be significant in capillary rise $\left(R_{c}\right)$ was proposed by Hamraoui and Nylander [9] and was calculated to be $2.5 \mathrm{~mm}$ for the oil used, which is much larger than the typical void/ window sizes in the foam. The capillary number $\left(C_{a}\right)$ was calculated to be on the order of $10^{-3}$ which is obviously the dominant force compared with gravity and inertia.

$B_{o}=\frac{\left(\rho-\rho_{\text {air }}\right) g D^{2}}{\gamma}=4 \times 10^{-5}$

$W_{e}=\frac{\rho v^{2} R}{\gamma}=4 \times 10^{-7}$

$R_{c}=2 \frac{\left(\gamma \cos \theta \mu^{2} \rho^{2} g^{3}\right)^{0.2}}{\rho g}=0.0025 \mathrm{~m}$

$C_{a}=\frac{\mu v}{\gamma}=5 \times 10^{-3}$

Given these results, the standard L-W analysis should be applicable to capillary imbibition within polyHIPE foams. Thus, the mass imbibed $(m)$ is predicted to be given by: 
$m^{2}=\frac{\rho^{2} A^{2} \phi^{2}\left(S_{w f}-S_{w i}\right)^{2} a^{3} r_{e f f} \gamma \cos \theta}{2 \mu \tau^{2}} t$

where $\rho$ is the liquid density, $A$ the cross sectional area, $\phi$ the foam porosity, $S_{w f}$ the foam saturation by the infiltrating liquid in volume percent, $S_{w i}$ is the initial saturation, $\mu$ the viscosity of the liquid, $\gamma$ the liquid surface tension, and $\theta$ the contact angle between the liquid and solid. Also, $r_{\text {eff }}$ is the effective average radius of the flow channels in which the imbibition occurs.

\subsubsection{Silicone oil imbibition}

The excellent wetting of the polymer polyHIPE foam material by the silicone oil is demonstrated by observation of the rapid spreading of a liquid drop on the flat polymer substrate (prepared by bulk polymerization of the monomer mixture) as shown in Fig. 6 . The vanishing contact angle demonstrated that silicone oil wets the polymer material perfectly. This is consistent with the fact that the silicone oil used here has a surface tension of $21 \mathrm{mN} / \mathrm{m}$ (measured by the Wilhelmy plate method) while the polymer has a reported surface energy of $30 \mathrm{mN} / \mathrm{m}$ [29].

Typical capillary imbibition data for silicone oil into a polyHIPE foam are shown in Fig. 7. The linear correlation between mass imbibed and the square root of time throughout the whole capillary rise process indicates that the flow behavior agrees with the standard assumptions in the $L-W$ analysis.

In the $L-W$ analysis, the structure of a porous media is depicted as an array of capillary tubes [3], the diameter of which determine the infiltration rates. The effective diameter for imbibition within the polyHIPE can be calculated using Eq. 15 [4] from the slope of the imbibition-rate curves. The results were found to be internally consistent between the three cases, with less than a $5 \%$ standard deviation between them.

To further test this result, polyHIPE foams with various window sizes (R10M05-M30) were tested with $100 \mathrm{cSt}$ silicone oil, which was chosen to enable experiments with a duration long enough for precision, but short enough to allow for multiple trials, with individual trials typically

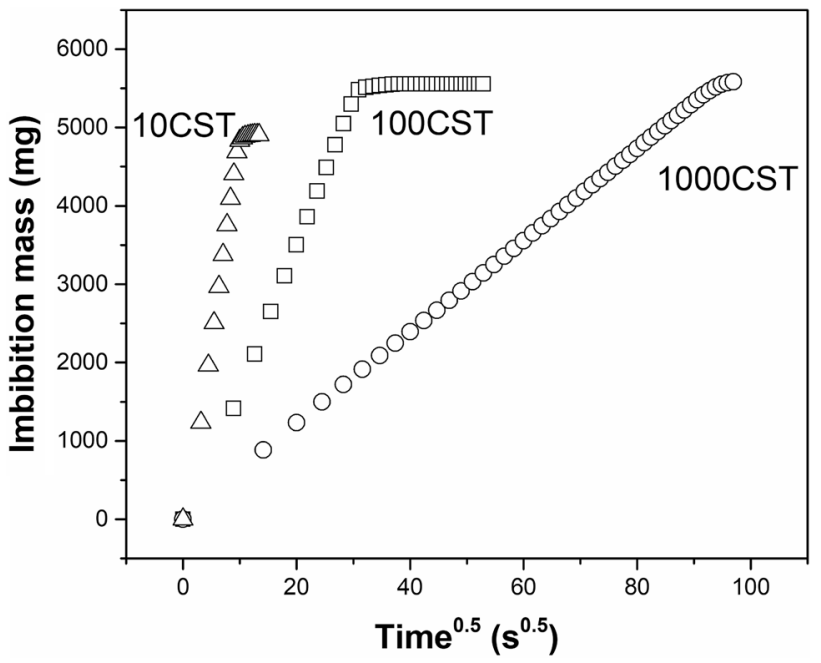

Fig. 7 Spontaneous imbibition test using three silicone oils with 10, 100 and 1000 cSt kinematic viscosity. The foam sample was R40M05

lasting 5-60 min. Figure 8 shows the effective diameter calculated from the imbibition results, and compares those results to the effective hydrodynamic diameters determined from the permeability tests, and the mean window size from the imaging studies. Interestingly, it was found that the effective hydrodynamic diameter matches more closely the window size rather than the void size, which suggests that the region of greatest hydrodynamic resistance (the windows) within the foam governs the imbibition process.

The degree of saturation indicated how much of the available pore space is filled by the penetrating liquid. None of the foams tested in this study showed a $100 \%$ saturation by the silicone oil. As shown in Fig. 9, the data suggests a correlation between saturation and the shearing time used during emulsion preparation. With an increase in the mixing time, the polydispersity of emulsion droplets decreases, as does the void and windows size. This leads to a more uniform penetration through the porous foam by the silicone oil. Although the macroscopic transport during imbibition is vertically upwards, there will

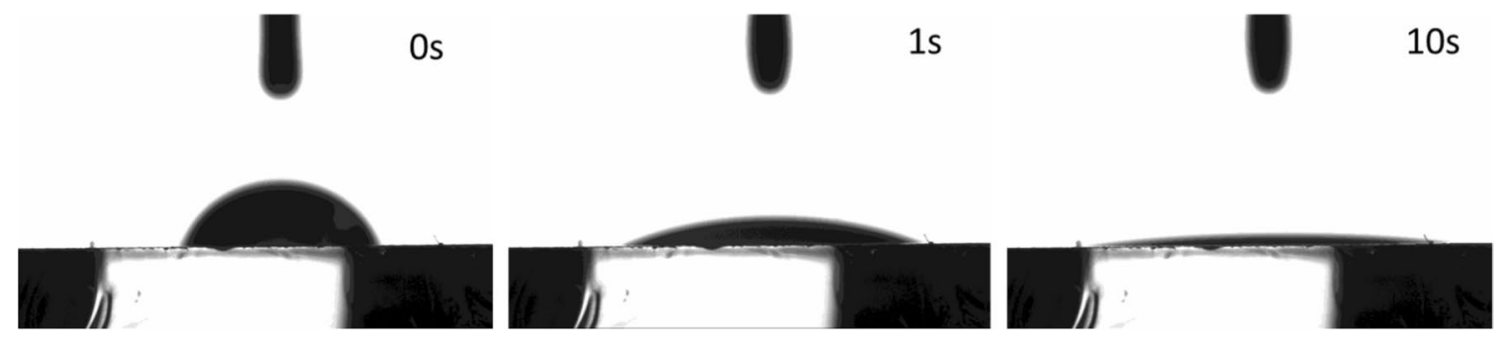

Fig. 6 Spreading of the silicone oil on the polymer substrate in bulk form 


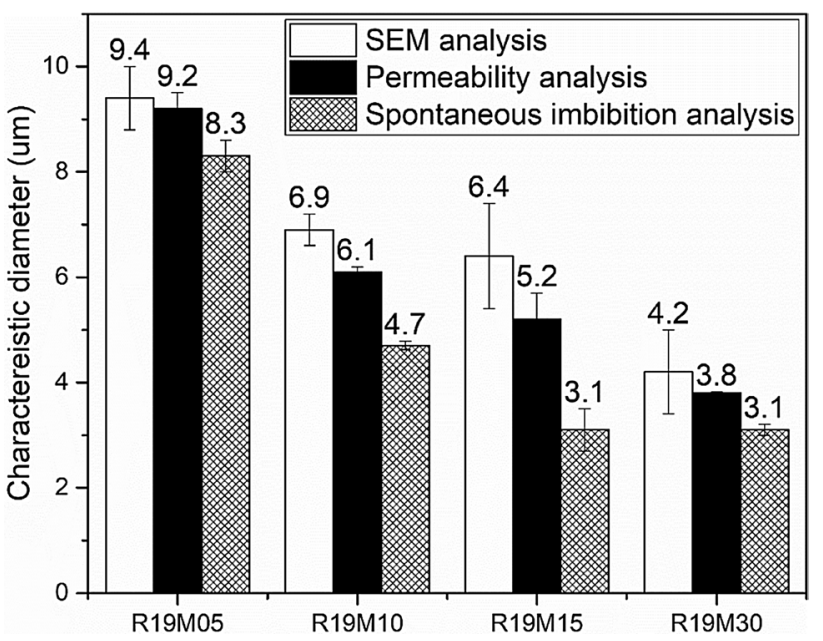

Fig. 8 Comparison of the effective hydrodynamic diameters from the imbibition and permeability studies with the mean window size determined from the imaging studies for four polyHIPE foams, R19M05, R19M10, R19M15, and R19M30

be transverse flow occurring due to the presence of many windows distributed around each void. This may lead to the entrapment of air (and correspondingly less than 100\% saturation) as is depicted schematically Fig. 10. The infiltrating liquid will preferentially pass through the larger channels (i.e., those voids with larger windows). This will eventually lead to a preferred flow path, and potentially trapping air pockets within the foam. Thus, the saturation data can be an indirect indication of the pore connectivity within the foam as experienced by the capillary driven flow.

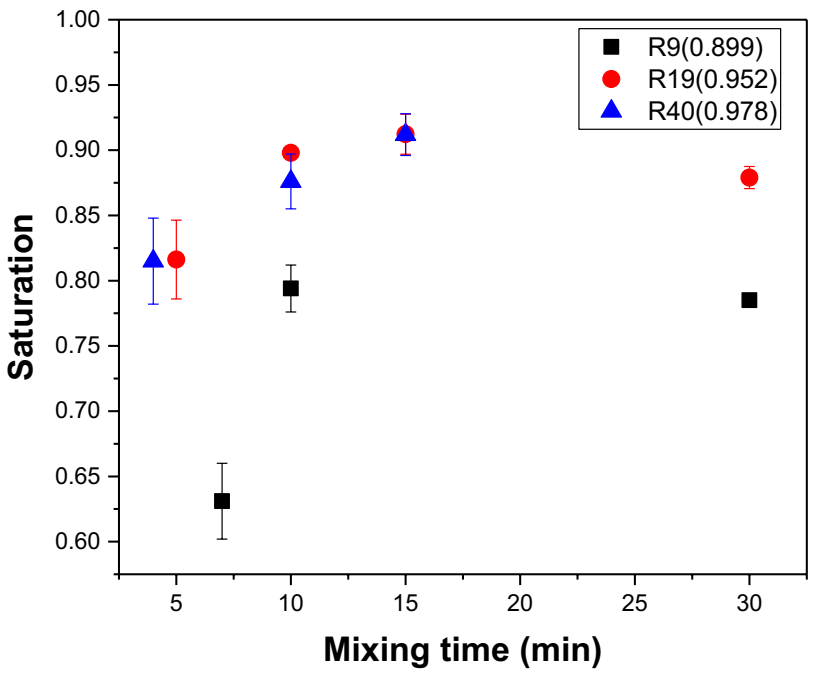

Fig. 9 Saturation data for silicone oil in foams prepared with various water-to-oil ratios and shearing times. The porosity of each series of foams is indicated (in parentheses) within the legend

\subsubsection{Partial wetting-water imbibition}

To study the imbibition of water, R19M10 foam was chosen because of the convenience of processing. Figure 11 shows the typical water spontaneous imbibition into R19M10 polyHIPE foams which were treated with various amounts of surfactant. The sample with no added emulsifier did not imbibe water. For all of the other samples, it was found that the slope of the imbibition curves gradually increased with time. During these experiments, it was observed that the water tends to penetrate into the foams faster near their periphery and slower near their center, as is illustrated in Fig. 12. The uneven imbibition front was found to continue developing until it reached the top surface of the sample. To minimize this effect, the thickness of the samples used in the imbibition tests was limited to $5 \mathrm{~mm}$ (sample diameter of $25 \mathrm{~mm}$ ).

Figure 13 shows the slope of the imbibition rate curves (known as the Washburn coefficients) as a function of the added surfactant content. The imbibition rate was found to increase with the surfactant content up to around $25 \mathrm{wt} \%$ but then decreases for higher concentrations. This result is attributed to the non-uniformity of the surfactant deposit within the foam. Thin polymer films under $100 \mathrm{~nm}$ may undergo dewetting on the substrate [22]. Given the internal surface area of the foams, and assuming a uniform layer of the surfactant on all of the internal surfaces, it is possible to compute an effective film thickness as a function of overall surfactant concentration. For a sample with $1.5 \mathrm{wt} \%$ emulsifier, a coating of $4 \mathrm{~nm}$ (and thus non-uniform deposits of the surfactant) would be expected. Increasing the surfactant concentration at low levels of surfactant should be expected to lead to better wettability. However, for $15 \mathrm{wt} \%$ surfactant added, the deposited layer would be $45 \mathrm{~nm}$. At these higher surfactant concentrations, deposits may block windows within the foam, thus leading to decreased imbibition rates.

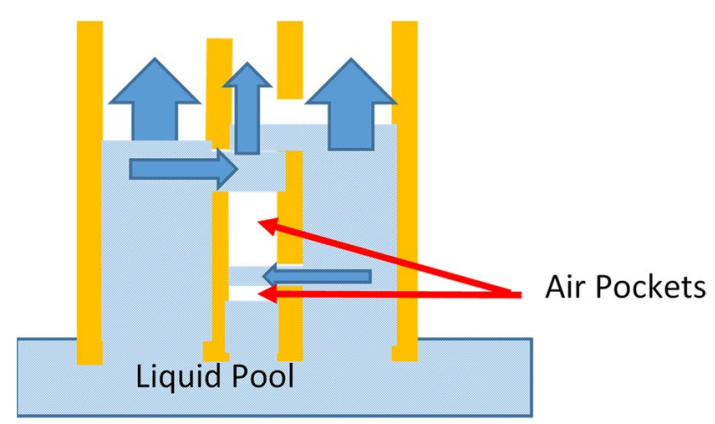

Fig. 10 Schematic depicting the entrapment of air during the imbibition process 
(a)

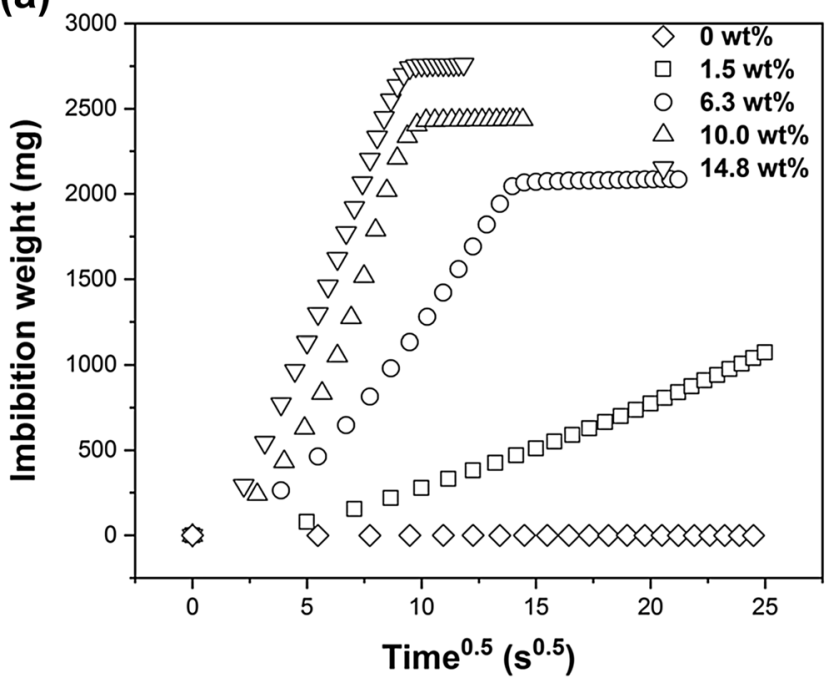

(b)

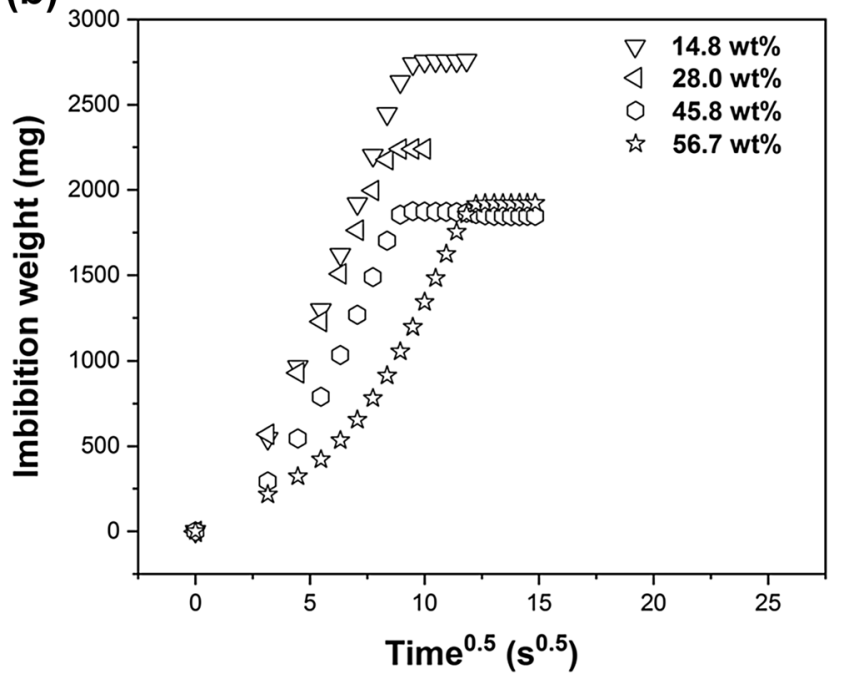

Fig. 11 Imbibition of water into R10M10 foams prepare with various surfactant content

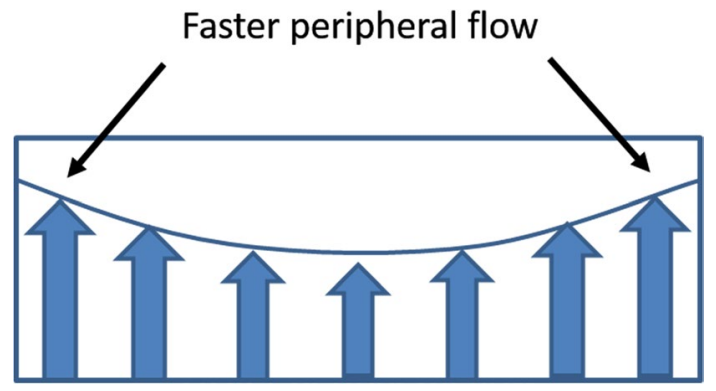

Fig. 12 Schematic illustrating the non-uniform infiltration front observed during the imbibition experiments

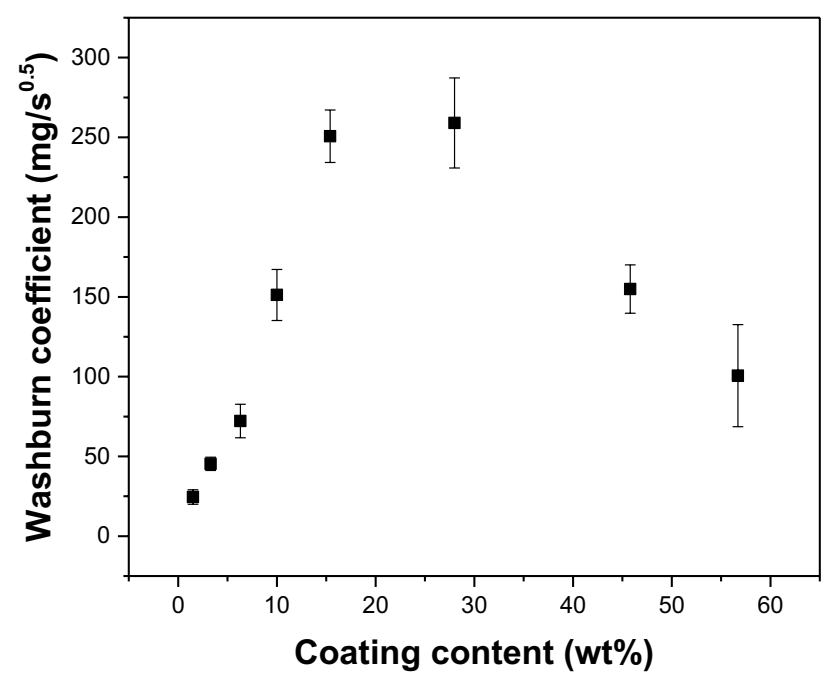

Fig. 13 Washburn coefficients resulting from the imbibition studies of water into foams with various surfactant contents

\section{Conclusions}

The droplet size within HIPEs can be controlled by varying the mixing time during the emulsification step, and thus the polyHIPE foams templated from the HIPEs can have a controlled void and window morphology.

For the permeability studies in the Darcy flow regime wherein the foams were fully saturated with the liquid, measurement of foams with various morphologies revealed that the window size is the characteristic length scale that determines the hydrodynamic resistance to flow.

In capillary-pressure driven infiltration, wetting of the foam polymer by the liquid plays a significant role: for liquids that spread on the polymer, the flow behavior in the present study is well described by the Lucas-Washburn law and reveals that the window size is the characteristic diameter governing fluid transport. However, as the polydispersity of the void size increases, the saturation of the foams decreases due to bypassing flow which entraps air pockets. For the partial wetting scenario, capillary infiltration behavior deviates from the Lucas-Washburn law because of the non-uniform surface wettability.

Author's Contribution All authors contributed to the study conception, design, and analysis. Material preparation, data collection and analysis were performed by Boran Zhao and Gabriel Gedler. The first draft of the manuscript was written by Boran Zhao and all authors commented on previous versions of the manuscript. All authors read and approved the final manuscript. 


\section{Compliance with ethical standards}

Conflict of interest On behalf of all authors, the corresponding author states that there is no conflict of interest.

\section{References}

1. Beeckman JW (1990) Mathematical description of heterogeneous materials. Chem Eng Sci 45(8):2603-2610

2. Bell JM, Cameron FK (1905) The flow of liquids through capillary spaces. J Phys Chem 10(8):658-674

3. Bico J, Thiele U, Quéré D (2002) Wetting of textured surfaces. Colloids Surf A 206(1-3):41-46

4. Cai J, Perfect E, Cheng C-L, Hu X (2014) Generalized modeling of spontaneous imbibition based on Hagen-Poiseuille flow in tortuous capillaries with variably shaped apertures. Langmuir 30(18):5142-5151

5. Cameron N, Sherrington D, Albiston L, Gregory D (1996) Study of the formation of the open-cellular morphology of poly (styrene/ divinylbenzene) polyHIPE materials by cryo-SEM. Colloid Polym Sci 274(6):592-595

6. Debye P, Cleland RL (1959) Flow of liquid hydrocarbons in porous vycor. J Appl Phys 30(6):843-849

7. Fries $N$, Dreyer $M(2008)$ The transition from inertial to viscous flow in capillary rise. J Colloid Interf Sci 327(1):125-128

8. Hager WH (2012) Wilfrid Noel Bond and the Bond number. J Hydraul Res 50(1):3-9

9. Hamraoui A, Nylander T (2002) Analytical approach for the Lucas-Washburn equation. J Colloid Interf Sci 250(2):415-421

10. Foudazi R, Gokun P, Feke DL, Rowan SJ, Manas-Zloczower I (2013) Chemorheology of Poly(high internal phase emulsions). Macromolecules 46(13):5393-5396

11. Grace HP (1982) Dispersion phenomena in high-viscosity immiscible fluid systems and application of static mixers as dispersion devices in such systems. Chem Eng Com 14(3-6):225-277

12. Gruner S (2010) Rheology and dynamics of simple and complex liquids in mesoporous matrices. Logos Verlag $\mathrm{GmbH}$, Berlin

13. Lake LW (1989) Enhanced oil recovery. Prentice Hall, Englewood Cliffs

14. Law YY, Feke DL, Manas-Zloczower I (2013) Method for probing the microstructure of particle beds using infiltration behavior. Powder Technol 237:427-431

15. Li K, Zhang D, Bian H, Meng C, Yang Y (2015) Criteria for applying the Lucas-Washburn law. Sci Rep 5:14085

16. Lissant KJ, Mayhan KG (1973) Study of medium and high internal phase ratio water-polymer emulsions. J Colloid Interf Sci 42(1):201-208
17. Lucas R (1918) Ueber das Zeitgesetz des kapillaren Aufstiegs von Flüssigkeiten. Kolloid-Zeitschrift 23(1):15-22

18. Moglia RS, Robinson J, Buffington S, Touchet T, Cosgriff-Hernandez E (2013) Fabrication of injectable and high porosity polyMIPE scaffolds for soft tissue regeneration. Abstr Pap Am Chem S 245 (2013)

19. Moglia RS, Whitely $M$, Dhavalikar $P$, Robinson J, Pearce $H$, Brooks M, Stuebben M, Cordner N, Cosgriff-Hernandez E (2014) Injectable polymerized high internal phase emulsions with rapid in situ curing. Biomacromol 15(8):2870-2878

20. Morrow NR (1990) Wettability and its effect on oil recovery. J Pet Technol 42(12):1476-1484

21. Pulko I, Smrekar V, Podgornik A, Krajnc P (2011) Emulsion templated open porous membranes for protein purification. J Chromatog A 1218(17):2396-2401

22. Reiter G (1992) Dewetting of thin polymer films. Phys Rev Lett 68(1):75-78

23. Robinson JL, Moglia RS, Stuebben MC, McEnery MAP, CosgriffHernandez E (2014) Achieving interconnected pore architecture in injectable PolyHIPEs for bone tissue engineering. Tissue Eng Pt A 20(5-6):1103-1112

24. Sevsek U, Brus J, Jerabek K, Krajnc P (2014) Post polymerisation hypercrosslinking of styrene/divinylbenzene poly(HIPE) $\mathrm{s}$ : creating micropores within macroporous polymer. Polymer 55(1):410-415

25. Still T, Yunker PJ, Yodh AG (2012) Surfactant-induced marangoni eddies alter the coffee-rings of evaporating colloidal drops. Langmuir 28(11):4984-4988

26. Taylor G (1934) The formation of emulsions in definable fields of flow. Proc R Soc Lond Ser A 146(858):501-523

27. Washburn EW (1921) The dynamics of capillary flow. Phys Rev 17(3):273-283

28. Williams JM, Wrobleski DA (1988) Spatial distribution of the phases in water-in-oil emulsions Open and closed microcellular foams from cross-linked polystyrene. Langmuir 4(3):656-662

29. Wu S (1971) Interfacial energetics and polymer adhesion. Org Coat Plast Chem 31:27

30. Xu H, Zheng X, Huang Y, Wang H, Du Q (2016) Interconnected Porous polymers with tunable pore throat size prepared via pickering high internal phase emulsions. Langmuir 32(1):38-45

31. Zhang T, Guo Q (2017) Continuous preparation of polyHIPE monoliths from ionomer-stabilized high internal phase emulsions (HIPEs) for efficient recovery of spilled oils. Chem Eng J 307:812-819

Publisher's Note Springer Nature remains neutral with regard to jurisdictional claims in published maps and institutional affiliations. 